\title{
Evidence of the factors that influence the utilisation of Kangaroo Mother Care by parents with low-birth-weight infants in low- and middle-income countries (LMICs): a scoping review protocol
}

\author{
Christina T. Mathias, Solange Mianda and Themba G. Ginindza
}

\begin{abstract}
Background: The Sustainable Development Goal (SDG) 3 emphasises on reducing neonatal deaths caused by low birth weight (LBW) complications by the implementation and utilisation of Kangaroo Mother Care (KMC) in low- and middle-income countries (LMICs). Despite the empirical evidence of KMC optimising low-birth-weight infants' (LBWIs') survival, its advantages and the LMICs implementing the service, studies have shown that LBW infant deaths occurring in LMICs are largely contributing to global child mortality. The aim of this scoping review is to map out the literature on barriers, challenges and facilitators of KMC utilisation by parents with LBWIs.
\end{abstract}

Methods and analysis: This scoping review will use Endnote $X 7$ reference management software to manage articles. The review search strategy will use SCIELO and LILACS databases. Other databases will be used via EBSCOHost search engine and these are Academic search complete, CINAHL with full text, Education source, Health source: Nursing/ Academic Edition, Medline with full text and Medline. We will also use Google Scholar, JSTOR, Open grey search engines and reference lists. A two-phase search mapping out process will be done. In phase 1, one reviewer will perform the title screening and removal of duplicates. Two reviewers will do a parallel abstract screening according to eligibility criteria. Phase 2 will involve the reading of full articles and exclusion of articles, in accordance with the eligibility criteria. Data extraction from the articles will be done by two reviewers independently and parallel to the data extraction form. The data quality assessment of the eligible studies will be done using the Mixed Method Appraisal Tool (MMAT). The extraction of the synthesised results and thematic content analysis of the studies will be done by NVIVO version 10.

Discussion: We expect to find studies on barriers, challenges and facilitating factors of KMC utilisation by parents with LBWIs in LMICs. The review outcomes will guide future research and practice and inform policy. The findings will be disseminated in print, electronic and conference presentations related to maternal child and neonatal health.

Keywords: Barriers, Challenges, Facilitating factors, Kangaroo Mother Care utilisation, Low-birth-weight infants, LMICs, Parents

\footnotetext{
* Correspondence: ctmathias@yahoo.ie

Discipline of Public Health Medicine, School of Nursing and Public Health,

University of KwaZulu-Natal, Durban, South Africa
} 


\section{Background}

\section{Global prevalence and impact of LBWI on neonatal} mortality

Kangaroo Mother Care (KMC), a skin-to-skin contact between the medically stable low-birth-weight infant (LBWI), weighing less than $2500 \mathrm{~g}$, and the parent's bare chest $[1,2]$, has proven to reduce LBWI mortality by almost 50\% [3-6]. Currently, low birth weight (LBW) mortality is a global leading cause of neonatal mortality contributing to $60-80 \%$ of neonatal deaths [7, 8]. Globally, an estimated 20 million low-birth-weight infants (LBWIs) are born annually, with low- and middle-income countries (LMICs) accounting for 18 million births [9]. LMICs bear a higher burden of LBWI outcomes due to the high prevalence of LBWI deaths as compared to high-income countries [3]. Each year, more than $50 \%$ of the LBWIs that are born in LMICs do not survive compared to their counterparts born in high-income countries [7].

\section{Management of LBWIs in LMICs and its outcomes}

The high LBWI deaths in LMICs are due to low economic income levels, poverty, poor health-seeking behaviour and weak health systems' links [7, 10]. As such in LMICs, the management of LBWI complications has been more of Kangaroo Mother Care as compared to conventional/incubator care, since around 1978 [4, 10-13]. LMICs strive to improve neonatal health through the implementation of KMC, among other interventions $[2,14]$, for instance, incorporating KMC package and guidelines in medical and nursing college syllabi, in-service training and existing national health care initiatives [1517]. However, although by 2015, 62 countries achieved the three-quarter child mortality reduction target, LBWI mortality contributed largely to child mortality deaths despite the LMICs implementing KMC [8, 18, 19]. Therefore, it is evident that KMC utilisation in LMICs is a challenge as LBWI mortality in LMICs still contributes largely to global neonatal and child mortality $[3,8,10,12,18]$.

\section{Problem statement and aim of the study}

The measure of success of KMC not only depends on KMC implementation but also on the utilisation of the service by the beneficiaries, parents with LBWIs [20, 21]. As such, the success of service utilisation largely depends on the utilisation determinants, absence of challenges and barriers, perceived quality of care, cost of care, supportive factors, cultural factors, health system factors and provider factors [20]. There are many studies focusing on KMC service delivery and healthcare providers; however, not many studies have focused on factors that influence $\mathrm{KMC}$ utilisation by parents with LBWIs [22]. World Health Organization (WHO) emphasises on employing strategies that will facilitate attaining the Sustainable Development Goal (SDG) 3, which aims at reducing newborn deaths to 12 neonatal deaths per 1000 live births per country [8, 10, 18]. In order to enhance achieving SDG 3, this scoping review, therefore, aims at mapping out existing literature on the factors influencing KMC utilisation by parents with LBWIs in LMICs. The objective of the study will be to identify and describe the factors influencing KMC utilisation by parents with LBWI in LMICs.

\section{Significance of the study}

This study will facilitate the identification of strategies/recommendations to inform policy development and/or update and inform future research and practice, by employing approaches to facilitate the uptake of KMC by parents with LBWIs.

\section{Methodology \\ Scoping review}

We plan to conduct a scoping of studies on barriers, challenges and facilitating factors of KMC utilisation by parents with LBWIs. The scoping review will facilitate the mapping out of new concepts, evidence-based knowledge and identified knowledge gaps [23]. The proposed study will adopt the framework by Arksey and O'Malley [24]. In summary, the framework involves the following:

i. Identifying the research question

ii. Identifying relevant studies

iii. Study selection

iv. Charting the data

v. Collating, summarising and reporting the results.

\section{Identifying the research question}

The research question is What is the documented evidence of the factors that influence the utilisation of KMC by parents with LBWI in LMICs? The research sub-questions are:

1. What are the facilitating factors of KMC utilisation by parents with LBWIs in LMICs?

2. What are the barriers and challenges to KMC utilisation by parents with LBWIs in LMICs? 
3. What are the experiences of $\mathrm{KMC}$ utilisation by parents with LBWIs in LMICs?

Eligibility of research question The amended SPIDER (Sample, Phenomenon of Interest, Design, Evaluation and Research type) framework will be used to determine the eligibility of the research question (see Table 1).

\section{Identifying relevant studies}

The scoping review will include qualitative, quantitative and mixed method primary research articles published in peer-reviewed journals and grey literature that address the research question. The review will include the following study designs: randomised control clinical trials, non-randomised experiments, survey, cross-sectional study designs, case control and cohort studies. The electronic databases that will be used to search for relevant articles will include Academic search complete, CINAHL with full text, Education source, Health source: Nursing/Academic Edition, Medline with full text and Medline. All these electronic databases will be accessed via EBSCOHost search engine. We will also search for studies from SCIELO and LILACS databases. Google Scholar search engine, JSTOR search engine, Open Grey search engine, 'the cited by' and reference lists will also be used to search for the relevant literature. Articles written only in English and those that can automatically be translated in English will be reviewed. The LMICs have been implementing KMC since it was introduced in 1978 by Ray; however, we will only include studies published between 1990 and 2017. The year 1990 was marked as the baseline for the United Nations development goals, as such that will be our starting point.

The search terms of this scoping review have originated from indexed subject headings, keywords of relevant studies, terms from this scoping review

Table 1 Framework determining eligibility of research question

\begin{tabular}{ll}
\hline Criteria & Determinant \\
\hline $\begin{array}{l}\text { Sample } \\
\begin{array}{l}\text { Phenomenon } \\
\text { of interest }\end{array}\end{array}$ & $\begin{array}{l}\text { Parents/guardian of LBWIs utilising KMC } \\
\text { Design }\end{array}$ \\
$\begin{array}{ll}\text { Randomised control clinical trials; non-randomised } \\
\text { experiments; survey; cross-sectional, case control and } \\
\text { cohort studies }\end{array}$ \\
$\begin{array}{l}\text { Evaluation } \\
\text { Research type }\end{array}$ & $\begin{array}{l}\text { Qnd facilitating factors to KMC utilisation } \\
\text { Qualive, quantitative and mixed method }\end{array}$ \\
\hline
\end{tabular}

protocol that recurred repetitively and the Medical Subject Headings (MeSH) terms. The string/Boolean search terms for this review will include kangaroo mother care OR kangaroo care OR skin to skin OR kangaroo-mother care method OR skin to skin contact AND parents OR mother OR father OR family caregivers AND low-birth-weight infants OR preterm infants OR premature infants OR very low birth weight infants AND utilization OR uptake OR compliance AND facilitators OR enablers OR motivators $\mathrm{OR}$ experience $\mathrm{OR}$ perception $\mathrm{OR}$ attitudes. The identified studies will be screened using eligibility criteria.

\section{Study selection}

The following criteria will guide the selection of studies.

Eligibility criteria Inclusion criteria

Studies meeting the following elements will be included in the study:

Studies written in English and in other languages with English version

Studies aiming at factors that influence the utilisation of KMC by parents of LBWIs in LMICs.

Studies focusing on experience/views/perception of parents with LBWIs on the utilisation of KMC in LMICs.

Studies with the above criteria and published between 1990 and 2017

Exclusion criteria

Studies with the following elements will be excluded from the study.

Studies written in other languages aside English; without English version

Studies with the above inclusion criteria but published before 1990

Studies with the above inclusion criteria but focusing on high-income countries

Studies with the above inclusion criteria but with incubator care as the phenomenon of interest whether in LMICs or high-income countries

Study selection procedure The selection of studies in this scoping review will involve two phases, as follows:

Phase 1

In phase 1 , one reviewer will perform title screening from the proposed databases, by examining the relevance of the study titles to the proposed research purpose. The identified articles will be imported to 
Table 2 Electronic search record

Keyword search Database used Number of studies retrieved

Endnote X7 reference management software, where duplicates will be removed. Table 2 will report the record of the number of articles identified on the electronic database.

The Endnote X7 library will then be shared with the two reviewers, who in parallel will independently screen the article abstracts according to the eligibility criteria. The full text of the eligible articles will be searched and kept in the EndNote X7 library. The reviewers will consult the University of KwaZulu-Natal Librarian to assist with the articles that will not have full text.

Phase 2

In phase 2, two reviewers will independently perform a parallel full article screening, following the eligibility criteria, and exclusion of articles with reasons. Data extraction will be performed on the eligible articles identified during the full article screening. Two reviewers will do the data extraction in parallel and independently, according to the data charting form as presented in Table 3. Notes will be shared between reviewers during abstract screening and full article screening. Where disagreements arise due to inclusion or exclusion of articles, the articles will be

Table 3 Data charting form

Author and date
Title of the study
Aim of the study/ research question
Population
Sample size
. \% and numberistics of participants
• Age/average of males
Intervention
Study design
Recruitment setting
Sampling strategy
Data collection (methodology)
Data analysis
Outcome of the study/results
Conclusion of the study
Significant findings
Comments

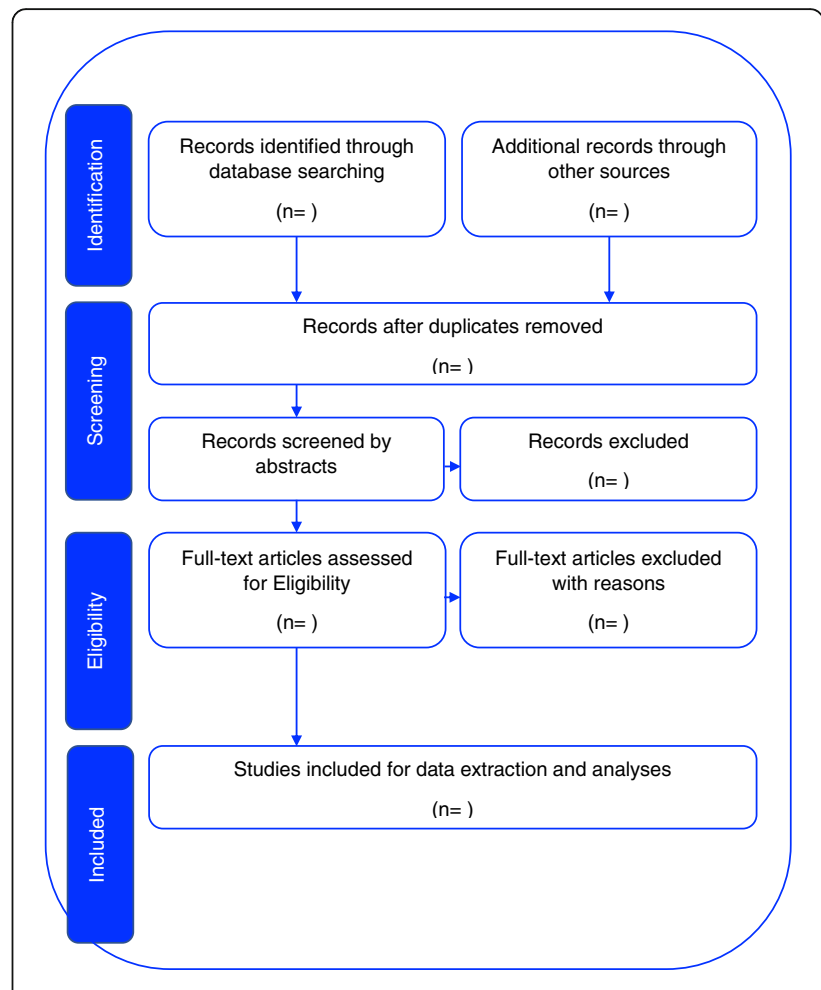

Fig. 1 Study selection process

sent to the third reviewer for reassessment and consideration.

Throughout the selection of eligible articles, the reviewer will keep account of the number of the articles imported to the Endnote X7 and the number of duplicates removed and keep account of the number of eligible articles for the abstract screening. During the abstract screening, the reviewers will take note of the number of excluded articles, indicating the reason for exclusion, and keep the number of articles eligible for full article screening. Reviewers for full article screening will also keep account of the number of articles excluded with reasons. The summary of the study selection process is shown in Fig. 1. The PRISMA-P checklist has been used to guide writing this scoping review protocol, as shown in Additional file 1. However, we will not assess the risk-of-bias assessment, which includes study-level bias, review-level bias and publication bias, as they are irrelevant to the nature of the scoping review [25].

The search strategy will be piloted to determine the validity and reliability of the criteria of the study selection. Table 4 in the Appendix narrates the pilot search results done on Medline via EBSCOHost search engine using the MeSH terms.

Charting the data 
We plan to use the elements included in the data charting form (see Table 3) to facilitate the standardised data extraction. The elements in the data charting form will be regularly updated, in order to address the research question.

\section{Collating, summarising and reporting the results}

The study outcomes that will guide data extraction will include challenges of KMC utilisation, barriers of KMC utilisation and facilitating factors of KMC utilisation. The thematic content analysis will be used to code arising themes and analyse the narrative account of the extracted data. The extracted data will be coded using NVIVO software version 10 [26]. Three stages will be used to guide collating, summarising and reporting of the results. The stages include coding text, developing descriptive themes and generating analytical themes [27]. However, these stages are interrelated in such a way that the free coding of the primary study findings facilitates the organisation of the codes into descriptive themes. The descriptive themes enhance the development of the analytical themes.

\section{Stages 1 and 2: Coding text and developing descrip-} tive themes In this review, two reviewers will independently and in parallel do the line-by-line coding of the primary study findings, in relation to the context and meaning. However, the coding of the study findings will not strictly depend on the research question, as there might be few studies addressing the review question directly [27]. The reviewers will then categorise the initial codes into major groups, depending on their similarities and differences, then new codes will be assigned to these grouped codes, in order to give a descriptive meaning to the groups. Hence, descriptive themes will be developed. One reviewer will write a draft summary of the descriptive themes, which will be reviewed by the rest of the reviewers and they will agree on the final version of the descriptive themes.

Stage 3: Generating analytical themes Independently and in parallel, the reviewers will deduce the barriers, challenges and facilitating factors of KMC utilisation by parents with LBWIs from the descriptive themes. In this stage, the reviewers will, through narration, analyse the descriptive themes and examine the relationship between themes to the review question. The reviewer will then examine the meaning of the study findings to the review question. The implications of the findings will be considered for intervention development. Through the narrative analysis process, individually, the reviewers will be able to develop analytical themes and proposed interventions. Then, the reviewers will together discuss the review question and the implications in relation to the descriptive themes. During the group discussion, we anticipate that more analytical themes and implications for intervention development will emerge. The process will be repeated until we no longer have emerging analytical themes and implications for intervention development. The reviewers will summarise stage 3 by agreeing and approving the identified analytical themes and implications for intervention development. The implications will form the recommendations of the review. Finally, the results will be reported.

\section{Quality appraisal}

The Mixed Method Appraisal Tool (MMAT) version 2011 will be used to assess the quality of the eligible studies in terms of the appropriateness of the study aim, the study design, methodology, sampling strategy, data collection, data analysis, result presentation, discussion and conclusion.

\section{Discussion}

This scoping review will form part of the study on 'Utilization of Kangaroo Mother Care in Mangochi district, Southern Malawi'. Mapping out the evidence that exists on barriers, challenges and facilitating factors of KMC utilisation in LMICs will provide evidence-based knowledge gaps, inform future research and enrich the main study's findings. The barriers and challenges to KMC utilisation that prevent parents with LBW infants from utilising the only low-cost effective measure in LMICs that manages LBW complications and prevents LBWIs' deaths will be identified. The outcomes of this scoping review will inform future research and identify the evidence-based interventions, which will inform policies and guidelines. This is in order to improve KMC utilisation in LMICs, prevent LBWI deaths and contribute towards the SDG 3 goal of 12 neonatal deaths per 1000 live births per country by 2030 [3, 8]. Although rigorous steps will be followed throughout this review, we anticipate some limitations. Firstly, studies may be omitted from the review if they were not published in the databases searched or if they were not published at all. Secondly, articles may not be accessible if they were published in languages other than English. 


\section{Appendix}

Table 4 Results of the pilot database search

\begin{tabular}{|c|c|c|c|}
\hline Keyword search & Date of search & Search engine used & $\begin{array}{l}\text { Number of } \\
\text { retrieved studies }\end{array}$ \\
\hline $\begin{array}{l}\text { (MM "Kangaroo-Mother Care Method/UT") AND (MM "Infant, Very } \\
\text { Low Birth Weight+") AND (MM "Infant, Low Birth Weight+/GD") } \\
\text { AND (MM "Infant, Extremely Low Birth Weight/GD") AND (MM } \\
\text { "Mothers/PX") AND (MM "Infant Care+/MT") Date of publication: } \\
\text { 19900101-20171231 } \\
\text { Source types: Academic Journals }\end{array}$ & 13 February 2018 & Medline via EBSCOHost & 976 \\
\hline
\end{tabular}

\section{Additional file}

Additional file 1: The PRISMA-P 2015 checklist. (DOCX 26 kb)

\section{Abbreviation}

KMC: Kangaroo Mother Care; LBW: Low birth weight; LBWI: Low-birth-weight infant; LBWIs: Low-birth-weight infants; LMICs: Low- and middle-income countries; MMAT: Mixed Method Appraisal Tool; SDGs: Sustainable Development Goals; SPIDER: Sample, Phenomenon of Interest, Design, Evaluation and Research type; UKZN: University of KwaZulu-Natal; WHO: World Health Organization

\section{Acknowledgements}

The authors acknowledge the University of KwaZulu-Natal (UKZN), College of Health Science, for the provision of resources towards the development of this scoping review protocol. The authors extend their gratitude to the following: the UKZN Subject Librarian: Nursing and Public Health, Nkululeko Magwaza, for assisting with the search term strategy and Professor Myra Taylor for assisting with editing this document

\section{Funding}

This study has been funded by the University of KwaZulu-Natal, College of Health Sciences Doctoral Research Scholarship Grant. The authors declare that the funders had no role in developing this protocol.

\section{Availability of data and materials}

The published scoping review study will contain all the generated and analysed data gathered during this study, for open access.

\section{Authors' contributions}

CTM conceptualised the study and drafted the study proposal under the supervision of SM and TGG. CTM, SM and TGG contributed to the development of the study background, design, methodology, the outcomes to guide data extraction and method of data synthesis. CTM prepared the manuscript and SM and TGG supervised and reviewed it. CTM, SM and TGG reviewed the final version of the protocol and approved it

\section{Ethics approval and consent to participate}

Not applicable

\section{Consent for publication}

Not applicable

\section{Competing interests}

The authors declare that they have no competing interests.

\section{Publisher's Note}

Springer Nature remains neutral with regard to jurisdictional claims in published maps and institutional affiliations.
Received: 6 September 2017 Accepted: 20 March 2018

Published online: 05 April 2018

\section{References}

1. World Health Organization. Kangaroo mother care: a practical guide. WHO Reproductive Health and Research. 2003:1-48. http://www.who.int/maternal_ child adolescent/documents/9241590351/en/. Accessed 20 June 2017.

2. World Health Organization. WHO recommendations on interventions to improve preterm birth outcomes. WHO. 2015:1-98. http://www.who.int/ reproductivehealth/publications/maternal_perinatal_health/preterm-birthguideline/en/\#. Accessed 9 Aug 2017.

3. UNICEF, World Health Organization, World Bank, UN-DESA Population Division. Levels and trends in child mortality. 2015. WHO. New York Ciy: World Health Organization. http://www.who.int/maternal_child_ adolescent/documents/levels_trends_child_mortality_2015/en/\#. Accessed 10 July 2017.

4. March of Dimes, PMNCH, Save the Children WHO. In: Howson CP, Kinney MV, Lawn JE, editors. Born too soon. The global action report on preterm birth, vol. 5. Geneva: World Health Organization Publ; 2012. p. 1-126. http:// www.who.int/pmnch/media/news/2012/201204_borntoosoon-report.pdf. Accessed 15 July 2017

5. Lawn JE, Mwansa-Kambafwile J, Horta BL, Barros FC, Cousens S. "Kangaroo mother care" to prevent neonatal deaths due to preterm birth complications (Structured abstract). Int J Epidemiol. 2010;2(1):i144-i154. https://doi.org/10.1093/ije/dyq03

6. Conde-Agudelo A, Díaz-Rossello JL. Kangaroo mother care to reduce morbidity and mortality in low birthweight infants. Cochrane Database Syst Rev. 2016:8:CD002771. https://doi.org/10.1002/14651858.CD002771.pub3.

7. World Health Organization. WHO | Preterm birth. WHO. World Health Organization. 2016. http://www.who.int/mediacentre/factsheets/fs363/en/. Acessed 17 Aug 2017

8. World Health Organization. Health in 2015: from MDGs, Millennium Development Goals to SDGs, Sustainable Development Goals. World Health Organization. 2015;204. http://who.int/gho/publications/mdgs-sdgs/en/. Accessed 30 July 2017

9. World Health Organization. WHO | Care of the preterm and/or low-birthweight newborn WHO. Geneva: World Health Organization; 2014. p. 1. http://www.who.int/maternal_child_adolescent/topics/newborn/care_of_ preterm/en/. Accessed 14 Aug 2017.

10. Kinney MV, Kerber K, Black RE, Cohen B, Nkrumah F, Coovadia H, et al. SubSaharan Africa's mothers, newborns, and children: where and why do they die? PLOS Med. 2010;7(6):e1000294.

11. World Health Organization. Children: reducing mortality. World Health Organization. 2016. http://www.who.int/mediacentre/factsheets/fs178/en/. Accessed 15 Oct 2017

12. Lawn JE, Blencowe H, Oza S, You D, Lee ACC, Waiswa P, et al. Every newborn: progress, priorities, and potential beyond survival. Lancet. 2014; 384(9934):189-205. https://doi.org/10.1016/S0140-6736(14)60496-7

13. Darmstadt GL, Bhutta ZA, Cousens S, Adam T, Walker N, de Bernis L, et al. Evidence-based, cost-effective interventions: how many newborn babies can we save? Lancet. 2005;365(9463):977-88. https://doi.org/10.1016/S01406736(05)71088-6

14. Victora CG, Requejo JH, Barros AJD, Berman P, Bhutta Z, Boerma T, et al. Countdown to 2015: a decade of tracking progress for maternal, newborn, and child survival. Lancet. 2016:387(10032):2049-59. https://doi.org/10.1016/ S0140-6736(15)00519-X

15. Zimba E, Kinney M, Kachale $F$, Waltensperger $K$, Blencowe $H_{4}$ Colbourn $T$, George J, et al. Newborn survival in Malawi: a decade of change and future 
implications. Health Policy Plan. 2012;27(3):iii88-iii103. https://doi.org/10. 1093/heapol/czs043S.

16. World Health Organization. Countdown to 2015 decade report (2000-2010) with country profiles: taking stock of maternal, newborn and child survival. Matern Newborn Child Surviv Countdown to 2015. World Health Organization. 2015;53. http://www.who.int/maternal_child_adolescent/ documents/9789241599573/en/. Accessed 10 Sep 2017.

17. Bergh A-M, Charpak N, Ezeonodo A, Udani RH, van Rooyen E. Education and training in the implementation of kangaroo mother care. South African J Child Heal. 2011;6(I 20):38-45. http://www.sajch.org.za/index.php/SAJCH/ article/view/417/297. Accessed 20 Aug 2017.

18. World Health Organization. From MDGS to SDGS: a new era for global public health 2016-2030. World Health Organization. 2015. http://www. who.int/mediacentre/events/meetings/2015/MDGs-SDGs-Summary.pdf. Accessed 7 Sep 2017.

19. World Health Organization/ WHO. WHO | Preterm birth [Internet]. WHO. World Health Organization. 2016. http://www.who.int/mediacentre/ factsheets/fs363/en/. Accessed 9 Aug 2017.

20. Shengelia B, Murray CJ, Adams OB. Beyond access and utilization: defining and measuring health system coverage. In Health systems performance assessment. Debates, methods and empiricism. World Health Organization. 2003;221-234. https://www.google.co.za/search?tbo=p\&tbm=bks\&q=isbn: 9241562455. Accessed 20 July 2017.

21. Vesel L, Bergh A-M, Kerber KJ, Valsangkar B, Mazia G, Moxon SG, et al. Kangaroo mother care: a multi-country analysis of health system bottlenecks and potential solutions. BMC Pregnancy Childbirth. 2015;15(S2): 1-16. https://doi.org/10.1186/1471-2393-15-S2-S5

22. Nyqvist KH. Given the benefits of kangaroo mother care, why has its routine uptake been so slow? Acta Paediatr. 2016;105(4):341-2. http://doi.wiley.com/ 10.1111/apa.13236

23. Colquhoun HL, Levac D, O'Brien KK, Straus S, Tricco AC, Perrier L, Kastner M, Moher D. Scoping reviews: time for clarity in definition, methods, and reporting. J Clin Epidemiol. 2014;67(12):1291-4. https://doi.org/10.1016/j. jclinepi.2014.03.013

24. Arksey H, O'Malley L. Scoping studies: towards a methodological framework. Int J Soc Res Methodol. 2005;8(1):19-32. https://doi.org/10.1080/ 1364557032000119616

25. Li G, Mbuagbaw L, Samaan Z, Jin Y, Nwosu I, Levine MAH, et al. State of reporting of primary biomedical research: a scoping review protocol. BMJ Open. 2017;7(3):e014749. https://doi.org/10.1136/bmjopen-2016-014749

26. Castleberny A. NVivo 10 [software program]. Version 10. QSR International; 2012. Am J Pharm Educ. 2014;78(1):25. https://doi.org/10.5688/ajpe78125.

27. Thomas J, Harden A. Methods for the thematic synthesis of qualitative research in systematic reviews. BMC Med Res Methodol. 2008;8(1):45. https://doi.org/10.1186/1471-2288-8-45

\section{Submit your next manuscript to BioMed Central and we will help you at every step:}

- We accept pre-submission inquiries

- Our selector tool helps you to find the most relevant journal

- We provide round the clock customer support

- Convenient online submission

- Thorough peer review

- Inclusion in PubMed and all major indexing services

- Maximum visibility for your research

Submit your manuscript at www.biomedcentral.com/submit

) Biomed Central 